\title{
PAPILIONOIDEA E HESPERIOIDEA (LEPIDOPTERA) DE CURITIBA E SEUS ARREDORES, PARANÁ, BRASIL, COM NOTAS TAXONÔMICAS SOBRE HESPERIIDAE ${ }^{1}$
}

\author{
Carlos G.C. Mielke ${ }^{2}$
}

\begin{abstract}
PAPILIONOIDEA AND HESPERIOIDEA (LEPIIOPTERA) FROM CURITIBA AND vicinity, Paraná, Brazil with taxonomic notes on Hesperiddae. A list of butterflies from Curitiba and vicinity, Paraná, Brazil is given. 486 species are listed. The following are syu.n.: Phanes hoffmani Bell, 1940 of Phanis tavola Schaus, 1902; Parphorus nemortus Bell, 1941 and Tigasis altona Evans, 1955 of Phlebodesfartuga Schaus, 1902; Megistias gisparoides Hayward, 1934 of Megistias sancoya Schaus, 1902; Hesperia curassavica Snellen, 1887 of Hesperia clavus Erickson, 1848. The following is a stat.n.: Vehilius almoneus vetus Mielke, 1969. The following are comb.n.: Phanes tavola (Schaus, 1902); Parphorus fartuga (Schaus, 1902); Cobalopsis sancoya (Schaus, 1902); Arita polistion (Schaus, 1902). The following is sp.rev.: Arita polistion (Schaus, 1902).

KEY WORDS. Butterflies, Curitiba, Paraná, Brazil
\end{abstract}

BIEZANKO (1938) foi o único autor até a presente data a publicar um artigo sobre as borboletas e mariposas de Curitiba e seus arredores, enumerando 25 espécies de lepidópteros diurnos.

Após esta publicação, muitas coletas foram realizadas, principalmente por Olaf H.H. Mielke, Mirna M. Casagrande e pelo próprio autor, possibilitando uma complementação e atualização desta relação, perfazendo um total de 486 espécies, contribuindo assim para estudos biogeográficos e de biodiversidade (LAMAS 1981; BROWN JR. 1987; EMMEL \& AUSTIN 1990; Lamas et al. 1991; MielKe \& CASAGRANDE 1992).

Das 25 espécies, anteriormente registradas, confirma-se 22. Phoebis argante argante (Fabricius, 1775) provavelmente foi citada para as regiões próximas ao município de Araucária, as quais não se incluem neste trabalho. Hypoleria adasa (Hewitson, 1855) não foi encontrada; supõe-se que Biezanko a tenha confundido com outras espécies semelhantes que ocorrem nesta região. E, finalmente, Protographium asius (Fabricius, 1781) (mencionado como Papilo assius sic) é uma espécie típica no Paraná de locais com no máximo $600 \mathrm{~m}$ de altitude e portanto, não deve ocorrer na área estudada.

1) Contribuição número 809 do Departamento de Zoologia, Universidade Federal do Paraná.

2) Departamento de Zoologia, Universidade Federal do Paraná, Caixa Postal 19020, 81531-970 Curitiba, Paraná, Brasil. Bolsista do CNPq. 


\section{MATERIAL E MÉTODOS}

A região de Curitiba e arredores está situada entre $49^{\circ} 22^{\prime}$ e $49^{\circ} 07^{\prime} \mathrm{W}$ e $25^{\circ} 18^{\prime}$ e $25^{\circ} 33^{\prime} \mathrm{S}$. O clima, no sistema de Koeppen enquadra-se como Cfb, ou detalhadamente, é subtropical, úmido mesotérmico, de ventos fracos e com ocorrência de geadas severas e frequentes, sem estação seca. A temperatura e a pluviosidade média anual estão em torno de $16,5^{\circ} \mathrm{C}$ e $1450 \mathrm{~mm}$, respectivamente (IPPUC 1991).

O relevo mostra-se levemente ondulado, com formação de colinas, morros baixos e terraços com altitude variando entre 850 e 1000m (IPPUC 1991).

A vegetação nativa foi drasticamente alterada pela ação do homem através de queimadas e devastações, sendo esta a razão pela qual inclui-se os arredores, sendo estes lugares próximos aos limites da cidade nos municípios de Almirante Tamandaré, Campo Largo, Colombo, Piraquara e São José dos Pinhais onde ainda se encontra esta vegetação. Embora a vegetação tenha sofrido a ação antrópica, o conjunto fitofisionômico permanece o mesmo. Segundo KLEIN \& HaTsCHBACH (1962) este conjunto pode ser dividido em cinco grupos: reflorestamentos de bracatinga; campos naturais e edáficos, bem como capões que se encontram entre os mesmos; matas de galeria e pequenos núcleos de floresta nativa. Somente foram coletados lepidópteros nos campos, nos capões, nas matas de galeria e nas pequenas florestas.

Para fins de levantamento utilizou-se a coleção do Departamento de Zoologia da Universidade Federal do Paraná e as coleções particulares de Olaf H.H. Mielke e do autor.

Na identificação dos exemplares utilizou-se as obras de SEITz (1924), D’ABrera (1981, 1984, 1987a,b e 1988), BROWN JR. (1992) e as nas coleções mencionadas.

\section{RESULTADOS}

A lepidopterofauna estudada está distribuída em duas superfamílias, seis famílias e 18 subfamílias, com seus respectivos número de espécies: Hesperioidea -Hesperiddae 210: Pyrrhopyginae 12; Pyrginae 58; Hesperiinae 136; Eumesiinae 4. Papilionolidea -Lycaenidae 45; -Riodinidae 40; -NymphalidaE 149: Heliconiinae 23, Nymphalinae 17, Limenitidinae 31, Charaxinae 9, Apaturinae 3, Morphinae 4, Brassolinae 12, Satyrinae 29, Danainae 5, Ithomiinae 15, Libytheinae 1; -PAPILIONIDAE 13: Papilioninae 13; -PIERIDAE 29: Dismorphiinae 6, Pierinae 23.

\section{HESPERIIDAE}

Pyrrhopyginae

1. Elbella mariae (Bell, 1931)

2. Elbella adonis (Bell, 1931)

3. Hegesippe hegesippe (Mabille \& Boullet, 1908) 
4. Pyrrhopyge charybdis charybdis Westwood, [1852]

5. Granila paseas (Hewitson, 1857)

6. Croniades machaon (Westwood, [1852])

7. Sarbia antias (Felder, 1859)

8. Sarbia pertyi (Ploetz, 1879)

9. Sarbia damippe Mabille \& Boullet, 1908

10. Sarbia xanthippe xanthippe (Latreille, [1824])

11. Sarbia sp.

12. Oxynetra roscius roscius (Hopffer, 1874)

\section{Pyrginae}

13. Phocides polybius phanias (Burmeister, 1880)

14. Phocides pialia pialia (Hewitson, 1857)

15. Phanus australis Miller, 1965

16. Epargyreus psendexadeus (Westwood, [1852])

17. Polygonus leo leo (Gmelin, 1790)

18. Polygonus manueli manueli Bell \& Comstock, 1948

19. Chioides catillus catillus (Cramer, [1779])

20. Aguna asander asander (Hewitson, 1867)

21. Typhedanus aziris (Hewitson, 1867)

22. Polythrix octomaculata octomaculata (Sepp, 1848)

23. Ridens fulima Evans, 1952

24. Urbanus proteus proteus (Linnaeus, 1758)

25. Urbanus pronta Evans, 1952

26. Urbanus esta Evans, 1952

27. Urbanus dorantes dorantes (Stoll, 1790)

28. Urbanus teleus Huebner, 1821

29. Urbanus procne (Ploetz, 1881)

30. Urbanus zagorus (Ploetz, 1881)

31. Urbanus albimargo rica Evans, 1952

32. Astraptes fulgerator fulgerator (Walch, 1775)

33. Astraptes naxos (Hewitson, 1867)

34. Astraptes erycina (Ploetz, 1881)

35. Astraptes alardus alardus (Stoll, 1790)

36. Astraptes elorus (Hewitson, 1867)

37. Autochton integrifascia (Mabille, 1891)

38. Autochton zarex (Huebner, 1818)

39. Celaenorrhinus eligius punctiger (Burmeister, 1878)

40. Celaenorrhinus sp. (prox. similis Hayward, 1933)

41. Oechydrus chersis evelinda (Butler, 1870)

42. Telemiades vespasius (Fabricius, 1793) 
43. Polyctor polyctor polyctor (Prittwitz, 1868)

44. Nisoniades bipuncta (Schaus, 1902)

45. Ocella monophthalma (Ploetz, 1884)

46. Viola minor (Hayward, 1933)

47. Viola violella (Mabille, 1897)

48. Staphylus coecatus (Mabille, 1891)

49. Diaeus lacaena lacaena (Hewiston, 1871)

50. Gorgythion begga begga (Prittwitz, 1868)

51. Pythonides lancea (Hewitson, 1868)

52. Milanion leucaspis (Mabille, 1878)

53. Xenophanes tryxus (Stoll, [1780])

54. Carrhenes canescens pallida Roeber, 1925

55. Anisochoria subpicta Schaus, 1902

56. Achlyodes busirus rioja Evans, 1953

57. Achlyodes mithridates thraso (Huebner, 1807)

58. Timochares trifascia trifascia (Hewitson, 1868)

59. Ebrietas anacreon anacreon (Staudinger, 1876)

60. Cycloglypha stellita Zikán, 1938

61. Helias phalaenoides palpalis (Latreille, [1824])

62. Theagenes dichrous (Mabille, 1878)

63. Chiomara asychis autander (Mabille, 1891)

64. Chiomara mithrax (Moeschler, 1879)

65. Erynnis funeralis (Scudder \& Burgess, 1870)

66. Pyrgus communis orcynoides (Giacomelli, 1928)

67. Pyrgus oileus orcus (Stoll, [1780])

68. Heliopetes omrina (Butler, 1870)

69. Heliopetes purgia Schaus, 1902

70. Heliopetes randa Evans, 1953

\section{Hesperiinae}

71. Levina levina (Ploetz, 1884)

72. Zariaspes mys (Huebner, 1808)

73. Anthoptus epictetus (Fabricius, 1793)

74. Corticea corticea (Ploetz, 1882)

75. Corticea noctis (Ploetz, 1882)

76. Corticea lysias potex Evans, 1955

77. Corticea mendica ssp.

78. Corticea oblinita (Mabille, 1891)

79. Corticea obscura Mielke, 1969

80. Corticea immocerina (Hayward, 1934)

81. Cantha ivea ivea Evans, 1955 
82. Vinius letis (Ploetz, 1883)

83. Vinius pulcherrimus Hayward, 1934

84. Callimormus interpunctata (Ploetz, 1884)

85. Callimormus beda (Ploetz, 1886)

86. Virga austrina (Hayward, 1934)

87. Virga riparia Mielke, 1969

88. Virga hygrophila Mielke, 1969

89. Sodalia coler (Schaus, 1902)

90. Artines satyr Evans, 1955

91. Artines sp.

92. Sabina sabina (Ploetz, 1882)

93. Lucida lucia lucia (Capronnier, 1874)

94. Lucida schmithi (Bell, 1930)

95. Lucida ranesus (Schaus, 1902)

96. Phanes tavola (Schaus, 1902), comb.n.

Phanis tavola Schaus, 1902. Proc. U.S. Nat. Mus. 24: 445; [holó]tipo macho, Trinidad, British West Indies; ${ }^{0}$ 6.027, National Museum of Natural History.

Phanes hoffmanni Bell, 1940. Amer. Mus. Novit. 1064: 4, fig. 5 (genitália masculina); holótipo macho, Nova Bremen [atualmente Dalbérgia], Santa Catarina, Brasil; coleção American Museum of Natural History.

Phanes hoffmanni Bell é syn.n. de Phanis tavola Schaus. O. Mielke examinou os holótipos. A procedência "Trinidad, British West Indies" do holótipo de tavola, certamente, é um lapso de etiquetagem.

97. Vidius vidius (Mabille, 1891)

98. Vidius similis Mielke, 1980

99. Vidius fido Evans, 1955

100. Vidius nappa Evans, 1955

101. Vidius mictra Evans, 1955

102. Igapophilus rufus Mielke, 1980

103. Nastra lurida (Herrich \& Schaeffer, 1869)

104. Monca branca Evans, 1955

105. Cymaenes tripunctatus tripunctatus (Latreille, [1824])

106. Cymaenes gisca Evans, 1955

107. Cymaenes odilia odilia (Burmeister, 1878)

108. Cymaenes perloides (Ploetz, 1882)

109. Cymaenes campestris Mielke, 1980

110. Vehilius inca (Scudder, 1872)

111. Vehilius clavicula (Ploetz, 1884)

112. Vehilius almoneus vetus Mielke, 1969, stat.n.

É uma subespécie de Vehilius almoneus Schaus, 1902. O holótipo de 
almoneus foi estudado por O. Mielke; está no National Museum of Natural History, Washington.

113. Sucova sucova (Schaus, 1902)

114. Mnasitheus ritans (Schaus, 1902)

115. Remella remus (Fabricius, 1798)

116. Moeris striga striga (Geyer, 1832)

117. Parphorus fartuga (Schaus, 1902), comb.n.

Phlebodes fartuga Schaus, 1902. Proc. U.S. Nat. Mus. 24: 445;

[holó]tipo macho, Nova Friburgo, Brasil, $\mathrm{n}^{0}$ 6.024; National Museum of Natural History.

Parphorus nemorus Bell, 1941. Amer. Mus. Novit. 1125: 6, fig. 13 (genitália masculina); holótipo macho, Nova Bremen [atualmente Dalbérgia], Santa Catarina, Brasil; American Museum of Natural History. Syn.n.

Tigasis altona Evans, 1955. Cat. Amer. Hesp. 5, p. 176, pl. 65 (genitália masculina); [holó]tipo macho; Alto da Serra, São Paulo, R. Spitz leg.; The Natural History Museum. Syn.n.

Parphorus nemorus Bell e Tigasis altona Evans são syn.n. de Phlebodes fartuga Schaus.

O. Mielke examinou os holótipos dos três.

118. Molla molla Evans, 1955

119. Papias phainis Godman, 1900

120. Cobalopsis nero (Herrich \& Schaeffer, 1869)

121. Cobalopsis miaba (Schaus, 1902)

122. Cobalopsis vorgia (Schaus, 1902)

123. Cobalopsis sancoya (Schaus, 1902), comb.n.

Megistias sancoya Schaus,1902. Proc. U.S. Nat. Mus. 24: 452;

[holó]tipo macho, Petrópolis, Brasil, $n^{\circ}$ 6.048; National Museum of

Natural History.

Megistias gisparoides Hayward, 1934. Rev. Soc. ent. arg. 6: 161, pl.

11, fig. 9 (ventral); holótipo fêmea, Iguazu, Misiones, Argentina; coleção Breyer [Museu de La Plata].- Mielke,1968; Atas. Soc. Biol.

Rio de Janeiro 12: 76; syn.: olivacea, prospa. Syn.n.

Lereodea olivacea Hayward, 1938. An. Soc. cient. arg. 126: 443, fig.

15 (genitália masculina); holótipo macho, El Dorado, Misiones, Argentina; coleção Hayward [Instituto Miguel Lillo].

Cobalopsis prospa Evans, 1955. Cat. Amer. Hesp. 4, p. 161, pl. 64

(genitália masculina); [holó]tipo macho, São Paulo, R. Spitz leg., The Natural History Museum.

Megistias gisparoides Hayward é syn.n. de Megistias sancoya Schaus.

O. Mielke examinou os holótipos.

124. Arita polistion (Schaus, 1902), sp.rev., comb.n.

Megistias polistion Schaus, 1902. Proc. U.S. Nat. Mus. 24: 450; 
[holó]tipo sem indicação de sexo, Petrópolis, Brasil, $\mathrm{n}^{\circ}$ 6.041; National Museum of Natural History, Washington, DC, USA.

É uma espécie de Arita Evans, 1955 muito próxima de A. serra Evans, 1955. O. Mielke estudou os holótipos de ambos, o segundo no The Natural History Museum, Londres, Inglaterra.

125. Lerema duroca lenta Evans, 1955

126. Cumbre cumbre (Schaus, 1902)

127. Cumbre belli belli (Hayward, 1939)

128. Psoralis stacura (Schaus, 1902)

129. Vettius artona (Hewitson, 1868)

130. Paracarystus evansi Hayward, 1938

131. Thoon circellata (Ploetz, 1882)

132. Justinia kora (Hewitson, 1877)

133. Eutychide physcella (Hewitson, 1866)

134. Lamponia lamponia (Hewitson, 1876)

135. Miltomiges cinnumomea (Herrich \& Schaeffer, 1869).

136. Dion meda (Hewitson, 1877)

137. Lychnuchus celsus (Fabricius, 1793)

138. Talides sergestus (Cramer, [1775])

139. Synale hylaspes (Stoll, [1781])

140. Lychnuchoides ozias ozias (Hewitson, 1878)

141. Perichares philetes aurina Evans, 1955

142. Perichares lotus (Butler, 1870)

143. Orses itea (Swainson, 1821)

144. Alera furcata Mabille, 1891

145. Lycas argentea (Hewitson, 1866)

146. Saturnus saturnus servus Evans, 1955

147. Quinta cannae (Herrich \& Schaeffer, 1869)

148. Quinta locutia (Hewitson, 1876)

149. Mucia zygia (Ploetz, 1886)

150. Decinea lucifer (Geyer, 1831)

151. Decinea percosius (Godman, 1900)

152. Cyclosma altama (Schaus, 1902)

153. Caligulana caligula (Schaus, 1902)

154. Orthos orthos hyalinus (Bell, 1930)

155. Conga chyduea (Butler, 1877)

156. Conga urqua (Schaus, 1902)

157. Conga iheringii (Mabille, 1891)

158. Conga immaculata (Bell, 1930)

159. Conga zela (Ploetz, 1883)

160. Ancyloxypha nitedula (Burmeister, 1878)

161. Copaeodes jean favor Evans, 1955 
162. Hylephila phyleus phyleus (Drury, [1773])

163. Hylephila ancora (Ploetz, 1883)

164. Polites vibex catilina (Ploetz, 1886)

165. Polites sp.

166. Wallengrenia premnas (Wallengren, 1860)

167. Pompeius pompeius (Latreille, [1824])

168. Pompeius dares (Ploetz, 1883)

169. Pompeius amblyspila (Mabille, 1898)

170. Anatrytone perfida (Möschler, 1879)

171. Euphyes leptosema (Mabille, 1898)

172. Euphyes fumata Mielke, 1972

173. Euphyes cherra Evans, 1955

174. Euphyes subferrugine'us biezankoi Mielke, 1972

175. Arotis sirene (Mabille, 1904)

176. Arotis derasa brunnea (Mielke, 1972)

177. Libra aligula decia (Hayward, 1948)

178. Libra anatolica (Ploetz, 1883)

179. Hansa devergens hydra Evans, 1955

180. Chalcone santarus (Bell, 1940)

181. Metron oropa (Hewitson, 1877)

182. Phemiades pohli pohli (Bell, 1932)

183. Lerodea eufala eufala (Edwards, 1869)

184. Calpodes ethlius (Stoll, 1782)

185. Panoquina ocola ocola (Edwards, 1863)

186. Panoquina hecebola (Scudder, 1872)

187. Panoquina sylvicola sylvicola (Herrich \&Schaeffer, 1865)

188. Zenis jebus jebus (Ploetz, 1882)

189. Zenis minos (Latreille, [1824])

190. Nyctelius nyctelius nyctelius (Latreille, [1824])

191. Nyctelius paranensis (Schaus, 1902)

192. Thespieus dalman (Latreille, [1824])

193. Thespieus jora Evans, 1955

194. Thespieus ethemides (Burmeister, 1878)

195. Thespieus aspernatus Draudt, 1923

196. Thespieus lutetia (Hewitson, 1866)

197. Thespieus xarina Hayward, 1948

198. Thespieus vividus (Mabille, 1891)

199. Thespieus catochra (Ploetz, 1882)

200. Niconiades merenda (Mabille, 1878)

201. Xeniades chalestra corna Evans, 1955

202. Thracides cleanthes cleanthes (Latreille, [1824]) 
203. Neoxeniades scipio scipio (Fabricius, 1793)

204. Pyrrhopygopsis socrates socrates (Ménétriés, 1855)

205. Gen. et sp. ign.

206. Gen. et sp. ign.

$$
\text { Eumesiinae (=Cyclopedinae, = Heteropterinae) }
$$

207. Dardarina rana Evans, 1955

208. Dardarina castra Evans, 1955

209. Dardarina jonesi Evans, 1955

210. Dardarina aspila Mielke, 1966

\section{RIODINIDAE}

1. Euselasia crinon Stichel, 1919

2. Euselasia hygenius occulta Stichel, 1919

3. Mesosemia odice (Godart, [1824])

4. Mesosemia friburgensis Schaus, 1902

5. Eurybia misellivestris Stichel, 1910

6. Napaea nepos orpheus (Westwood, [1851])

7. Napaea umbra (Boisduval, 1870)

8. Chorinea licursus (Fabricius, 1775)

9. Panara soana trabalis Stichel, 1916

10. Brachyglenis drymo (Godman \& Salvin, 1886)

11. Lepricornis sp.

12. Barbicornis basilis mona Westwood, [1851]

13. Syrmatia nyx (Huebner, 1817)

14. Calephelis braziliensis McAlpine, 1971

15. Charis cadytis cadytis Hewitson, 1866

16. Chalodeta theodora theodora (Felder, 1862)

17. Crocozona sp.

18. Bacotis hisbon (Cramer, [1775])

19. Riodina lycisca lycisca (Hewitson, 1852)

20. Melanis smithice (Westwood, [1851])

21. Mesene pyrippe sanguilenta Stichel, 1910

22. Symmachia arion (C. Felder \& R. Felder, 1865)

23. Pterographium satnius satnius (Dalman, 1823)

24. Stichelia bocchoris suavis (Stichel, 1911)

25. Stichelia dunkinfieldia (Schaus, 1902)

26. Anteros formosus (Cramer, [1777])

27. Aethius archytes (Stoll, 1787)

28. Emesis fatima (Cramer, [1780]) 
29. Emesis ocypore zelotes Hewitson, 1872

30. Emesis diogenia Prittwitz, 1865

31. Emesis satema (Schaus, 1902)

32. Emesis neemias Hewitson, 1872

33. Emesis russula Stichel, 1910

34. Emartugina bifasciata bifasciata (Mengel, 1902)

35. Lemonias ochracea (Mengel, 1902)

36. Lemonias albofasciata (Godman, 1903)

37. Adelotypa sejuncta (Stichel, 1910)

38. Aricoris tutana (Godart, [1824])

39. Synargis phillone (Godart, [1824])

40. Synargis regulus (Fabricius, 1793)

\section{LYCAENIDAE}

1. "Thecla" catrea Hewitson, 1874

2. "Thecla" phydela Hewitson, 1867

3. Allosmaitia strophius (Godart, 1821)

4. Arcas ducalis (Westwood, [1852])

5. Pseudolycaena marsyas marsyas (Linnaeus, 1758)

6. Atlides cosa (Hewitson, 1867)

7. Atlides sp. prox. cosa (Hewitson, 1867)

8. Theritas triquetra (Hewitson, 1865)

9. "Thecla" deniva Hewitson, 1874

10. Rekoa malina (Hewitson, 1867)

11. Thereus ortalus (Godman \& Salvin, 1887)

12. Arawacus meliboeus (Fabricius, 1793)

13. Arawacus bolima (Schaus, 1902)

14. Arawacus tadita (Hewitson, 1877)

15. Contrafacia imma (Prittwitz, 1865)

16. Contrafacia muattina (Schaus, 1902)

17. Chlorostrymon simcathis (Drury, 1770)

18. Cyanophrys herodotus (Fabricius, 1793)

19. Cyanophrys bertha (Jones, 1912)

20. Cyanophrys remus (Hewitson, 1868)

21. Parrhasius selika (Hewitson, 1874)

22. Olynthus fancia (Jones, 1912)

23. Michaelus jebus (Godart, 1822)

24. Strymon crambusa (Hewitson, 1874)

25. Strymon bazochii (Godart, 1822)

26. Strymon sp. prox. bazochii (Godart, 1822)

27. Strymon cydia (Hewitson, 1874) 
28. Strymon eurytulus (Huebner, 1819)

29. Strymon oreala (Hewitson, 1868)

30. Strymon sp. prox. legota (Hewitson, 1877)

31. Lamprospilus badaca (Hewitson, 1868)

32. "Thecla" nubilum Druce, 1907

33. Electrostrymon endymion (Fabricius, 1775)

34. Calycopis chacona (Joergensen, 1932)

35. "Thecla" elika Hewitson, 1867

36. "Thecla" torris Druce, 1907

37. "Thecla" xorema Schaus, 1902

38. "Thecla" vanessoides (Prittwitz, 1865)

39. Ministrymon azia (Hewitson, 1873)

40. Brangas silumena (Hewitson, 1867)

41. Nesiostrymon calchinia (Hewitson, 1868)

42. Erora sp. prox. campa (Jones, 1912)

43. Zizula cyna tulliola (Godman \& Salvin, 1887)

44. Leptotes cassius (Cramer, [1775])

45. Hemiargus hanno (Stoll, 1790)

\section{NYMPHALIDAE}

\section{Heliconiinae}

1. Actinote alalia (C. Felder \& R. Felder, 1860)

2. Actinote genitrix d'Almeida, 1922

3. Actinote mamita mitama (Schaus, 1902)

4. Actinote melanisans Oberthuer, 1917

5. Actinote paripheles Jordan, 1913

6. Actinote pellenea pellenea Huebner, [1821]

7. Actinote carycina Jordan, 1913

8. Actinote surima surima (Schaus, 1902)

9. Actinote sp. prox. alalia (C. Felder \& R. Felder, 1860)

10. Actinote sp. prox. alalia (C. Felder \& R. Felder, 1860)

11. Euptoieta claudia hortensia (Blanchard, 1852)

12. Philathria wernickei (Roeber, 1906)

13. Dione juno juno (Cramer, [1779])

14. Agraulis vanillae maculosa (Stichel, 1907)

15. Dryadula phactusa (Linnaeus, 1758)

16. Dryas iulia alcionea (Cramer, [1779])

17. Eueides isabella dianasa (Huebner, [1806])

18. Eueides pavana Ménétriés, 1857

19. Eueides aliphera aliphera (Godart, 1819) 
20. Heliconius besckei Ménétriés, 1857

21. Heliconius erato phyllis (Fabricius, 1775)

22. Heliconius ethilla narcaea Godart, 1819

23. Heliconius sara apseudes (Huebner, [1813])

\section{Nymphalinae}

24. Hypanartia bella (Fabricius, 1793)

25. Hypanartia lethe (Fabricius, 1793)

26. Vanessa carye (Huebner, [1812])

27. Vanessa myrinna (Doubleday, [1849])

28. Vanessa braziliensis (Moore, 1883)

29. Anartia anathea roeselia (Eschscholtz, 1821)

30. Anartia jatrophae jatrophae (Linnaeus, 1763)

31. Junonia evarete (Cramer, [1779])

32. Siproeta epaphus trayja Huebner, [1823]

33. Chlosyne lacinia saundersi (Doubleday, [1847])

34. Eresia lansdorfi (Godart, 1819)

35. Ortilia ithra (Kirby, 1900)

36. Ortilia orthia (Hewitson, 1864)

37. Ortilia velica velica (Hewitson, 1864)

38. Telenessa teletusa (Godart, [1824])

39. Tegosa claudina (Eschscholtz, 1821)

40. Tegosa orohia (Hewitson, 1864)

\section{Limenitidinae}

41. Biblis hyperia nectanabis (Fruhstorfer, 1909)

42. Catonephele numilia penthia (Hewitson, 1852)

43. Catonephele sabrina (Hewitson, 1852)

44. Eunica caelina caelina (Godart, [1824])

45. Eunica eburnea Fruhstorfer, 1907

46. Myscelia orsis (Drury, 1782)

47. Ectima thecla thecla (Fabricius, 1796)

48. Hanadryas amphinome amphinome (Linnaeus, 1767)

49. Hamadryas epinome (C. Felder \& R. Felder, 1867)

50. Hamadryas februa februa (Huebner, [1823])

51. Hamadryas fornax fornax (Huebner, [1823])

52. Hanadryas iphthime iphthime $(\mathrm{H} . \mathrm{W}$. Bates, 1864)

53. Epiphile huebneri Hewitson, 1861

54. Epiphile orea orea (Huebner, [1823])

55. Temenis laothoe meridionalis Ebert, 1965 
56. Dynamine myrrhina (Doubleday, [1849])

57. Dynamine mylitta mylitta (Cramer, [1779])

58. Dynamine tithia tithia (Huebner, 1823)

59. Dynamine agacles agacles (Dalman, 1823)

60. Callicore pygas eucale (Fruhstorfer, 1916)

61. Diaethria candrena candrena (Godart, [1824])

62. Diaethria meridionalis (H. W. Bates, 1864)

63. Diaethria eluina eluina (Hewitson, [1855])

64. Callidula pyrame pyrame Huebner, [1819]

65. Adelpha mythra (Godart, [1824])

66. Adelpha gavina Fruhstorfer, [1916]

67. Adelpha hyas radiata Fruhstorfer, [1916]

68. Adelpha zea (Hewitson, 1850)

69. Adelpha calliphana Fruhstorfer, [1913]

70. Adelpha syma (Godart, [1824])

71. Marpesia petreus petreus (Cramer, [1776])

\section{Charaxinae}

72. Consul fabius drurii (Butler, [1874])

73. Zaretis itys itylus (Westwood, [1850])

74. Memphis hirta (Weymer, 1907)

75. Memphis otrere (Huebner, [1825])

76. Archaeoprepona amphimachus pseudomeander (Fruhstorfer, 1906)

77. Archaeoprepona chalciope (Huebner, [1823])

78. Archaeoprepona demophon thalpius (Huebner, [1814])

79. Prepona proschion Fruhstorfer, 1904

80. Siderone galanthis ssp.

\section{Apaturinae}

81. Doxocopa kallina (Staudinger, 1886)

82. Doxocopa laurentia laurentia (Godart, [1824])

83. Doxocopa zunilda zunilda (Godart, [1824])

\section{Morphinae}

84. Cytheritis aega (Huebner, [1822])

85. Cytheritis portis portis (Hübner, [1821])

86. Iphixibia anaxibia (Esper, [1801])

87. Pessonia epistrophus catenaria (Perry, 1811) 


\section{Brassolinae}

88. Brassolis astyra Godart, [1824]

89. Penetes pamphanis Doubleday, [1849]

90. Narope cyllene C. Felder \& R. Felder, 1859

91. Dynastor napoleon Doubleday, [1849]

92. Blepolenis bassus (C. Felder \& R. Felder, 1867)

93. Blepolenis batea batea (Huebner, [1821])

94. Opsiphanis invirae amplificatus Stichel, 1904

95. Opoptera fruhstorferi (Roeber, 1896)

96. Opoptera sulcius (Staudinger, 1887)

97. Catoblepia amphiroe (Huebner, [1825])

98. Eryphanis reevesii (Doubleday, [1849])

99. Caligo martia (Godart, [1824])

\section{Satyrinae}

100. Eteona tisiphone (Boisduval, 1836)

101. Praepedaliodes phanias (Hewitson, 1862)

102. Psendocercyonis glaucope glaucope (C. Felder \& R . Felder, 1867)

103. Capronnieria galesus (Godart, [1824])

104. Erichthodes julia (Weymer, 1911)

105. Forsterinaria necys (Godart, [1824])

106. Forsterinaria quantius (Godart, [1824])

107. Godartiana muscosa (Butler, 1870)

108. Hermeuptychia hermes (Fabricius, 1775)

109. Moneuptychia soter (Butler, 1877)

110. Moneuptychia moneca (Schaus, 1902)

111. Paryphthimoides poltys (Prittwitz, 1865)

112. Paryphthimoides ambigua (Butler, 1867)

113. Paryphthimoides phronius (Godart, [1824])

114. Splendeuptychia hygina (Butler, 1877)

115. Taygetis ypthima Huebner, [1821]

116. Taygetis acuta Weymer, 1910

117. Yphthimoides castrensis (Schaus, 1902)

118. Yphthimoides ochracea (Butler, 1867)

119. Zischkaia pacarus (Godart, [1824])

120. "Euptychia" peculiaris Butler, 1874

121. "Euptychia" griseldis Weymer, 1911

122. "Euptychia" paeon (Godart, [1824])

123. "Euptychia" pronophila Butler, 1867

124. "Euptychia" ocelloides Schaus, 1902 
125. "Euptychia" periphas (Godart, [1824])

126. "Euptychia" sp.

127. "Euptychia" sp.

128. "Euptychia" sp.

\section{Danainae}

129. Ituna ilione ilione (Cramer, [1775])

130. Danaus eresimus plexaure (Godart, [1819])

131. Danaus gilippus gilippus (Cramer, [1775])

132. Danaus plexippus erippus (Cramer, [1779])

133. Lycorea halia discreta Haensch, 1909

\section{Ithomiinae}

134. Mechanitis lysimnia lysimnia (Fabricius, 1793)

135. Methona themisto (Huebner, 1818)

136. Epityches eupompe (Geyer, 1832)

137. Placidula euryanassa (C. Felder \& R. Felder, 1865)

138. Ithomia drymo Huebner, 1816

139. Callithomia lenea methonella (Weymer, 1875)

140. Dircenna dero rhoeo C. Felder \& R. Felder. 1860

141. Prittwitzia hymenaea hymenaea (Prittwitz, 1865)

142. Episcada carcinia Schaus, 1902

143. Episcada philoclea Hewitson, [1855]

144. Hyalenna pascua (Schaus, 1902)

145. Pteronymia sylvo (Geyer, 1832)

146. Pseudoscada erruca (Hewitson, 1855)

147. Thyridia psidii cetoides (Rosenberg \& Talbot, 1914)

148. Heterosais edessa (Hewitson, [1855])

\section{Libytheinae}

149. Libytheana carinenta carinenta (Cramer, [1777])

\section{PAPILIONIDAE}

Papilionidae

1. Protesilaus helios (Rothschild \& Jordan, 1906)

2. Protesilaus stenodesmus (Rothschild \& Jordan, 1906)

3. Mimoides lysithous lysithous (Huebner, [1821])

4. Battus polydamas polydamas (Linnaeus, 1758) 
5. Battus polystictus polystictus (Butler, 1874)

6. Parides agavus (Drury, 1793)

7. Parides bunichus bunichus (Huebner, [1821])

8. Pterourus menatius cleotas (Gray, 1832)

9. Pterourus scamander scamander (Boisduval, 1836)

10. Heraclides anchisiades capys (Huebner, [1809])

11. Heraclides astyalus astyalus (Godart, [1819])

12. Heraclides thoas brasiliensis (Rothschild \& Jordan, 1906)

13. Heraclides hectorides (Esper, 1794)

\section{PIERIDAE}

\section{Dismorphiinae}

1. Pseudopieris nehemia nehemia (Boisduval, 1836)

2. Dismorphia astyocha Huebner, [1821]

3. Dismorphia melia (Godart, [1824])

4. Dismorphia thermesia thermesia (Godart, 1819)

5. Dismorphia amphiona astynome (Dalman, 1823)

6. Enantia clarissa (Weymer, 1895)

\section{Pierinae}

7. Colias lesbia lesbia (Fabricius, 1775)

8. Anteos clorinde (Godart, [1824])

9. Anteos menippe (Huebner, [1818])

10. Phoebis neocypris neocypris (Huebner, [1823])

11. Phoebis philea philea (Linnaeus, 1763)

12. Phoebis sennae sennae (Linnaeus, 1758)

13. Rhabdodryas trite banksi (Brown, 1929)

14. Aphrissa statira statira (Cramer, [1777])

15. Pyrisitia lence leuce (Boisduval, 1836)

16. Eurema albula albula (Cramer, [1776])

17. Eurema arbela arbela Geyer, 1832

18. Eurema deva deva (Doubleday, [1847])

19. Eurema phiale paula (Roeber, 1909)

20. Hesperocharis erota (Lucas, 1852)

21. Hesperocharis lactea lactea (Burmeister, 1879)

22. Archonias tereas tereas (Godart, 1819)

23. Catasticta bithys (Huebner, [1831])

24. Pereute swainsoni (Gray, 1832)

25. Glutophrissa drusilla drusilla (Cramer, [1777])

26. Leptophobia aripa aripa (Boisduval, 1836) 
27. Tatochila autodice autodice (Huebner, 1818)

28. Theochila maenacte maenacte (Boisduval, 1836)

29. Ascia monuste orseis (Godart, 1819)

AGRADECIMENTOS. A Profa. Dra. Mirna Martins Casagrande pelas sugestões e revisão deste trabalho, bem como a identiticação dos Brassolinas. Aos Profs. Drs. Olaf H.H. Mielke, Curtis Callaghan e Robert Robbins pela identificação de alguns Hesperiidae, Riodinidae e Lycaenidae respectivamente.

\section{REFERÊNCIAS BIBLIOGRÁFICAS}

BELL, E.L. 1941. New species of neotropial Hesperiidae (Lepidoptera: Rhophalocera). Amer. Mus. Novit. 1125: 1-10.

BIEZANKO, C. 1938. Sobre alguns lepidópteros que ocorrem em arredores de

Curitiba (Estado do Paraná). Pelotas, Livraria do Globo, 8p.

BROWN JR., K.S. 1987. Zoogeografía da região do Pantanal Mato-Grossense. In: Anais do $1^{0}$ Simpósio sobre Recursos Naturais e Sócio-Econômicos do Pantanal, DDT, EMBRAPA, Brasília, p.137-178.

1992. Borboletas da Serra do Japi: diversidade, hábitats, recursos alimentares e variação temporal. In: L.P.C. MORELLATO (Ed.). História Natural da Serra do Japi. Ecologia e preservação de uma área florestal no Sudeste do Brasil. Campinas, UNICAMP/FAPESP, $321 \mathrm{p}$.

D'Abrera, B. 1981. Butterflies of the Neotropical Region. Part I. Papilionidae and Pieridae. East Melbourne, Australia, Lansdowne, 172p.

1984. Butterflies of the Neotropical Region. Part II. Danaidae, Ithomiidae, Heliconidae and Morphidae. Victoria, Australia, Hill House, p. 174-384.

1987a. Butterflies of the Neotropical Region. Part III. Brassolidae, Acraeidae and Nymphalidae (partim). Victoria, Australia, Hill House, p. 386-525.

1987b. Butterflies of the Neotropical Region. Part IV. Nymphalidae (partim). Victoria, Australia, Hill House, p.580-678.

- 1988. Butterflies of the Neotropical Region. part V. Nymphalidae (concl.) and Satyridae. Victoria, Australia, Hill House, p.680-877.

Emmel, T.C. \& G.T. Austin. 1990. The tropical rain forest butterfly fauna of Rondonia, Brasil: species diversity and conversation. Trop. Lep., Gainesville, $1(1): 1-12$.

Evans, W.H. 1955. A Catalogue of the American Hesperiidae in the British Museum (Natural History). London, Trustees of the British Museum, V+499p.

Godman, F.D. 1900. In: F.D. Godman \& O. Salvin. Biologia Centr.-Amer., Insecta, Lepidoptera, Rhopalocera. 2, p.461-588, 3, pls 92-100.

HAYWARD, K.J. 1934. Lepidopteros argentinos. Familia Hesperidae. IV Subfamilia Pamphilinae. V Resumen, Clave, Apéndices e Indice. Rev. Soc. ent. 
arg. 6: 97-233.

Hayward, K.J. 1938. Hesperioidea argentina VIII. An. Soc. cient. arg. 126: 429-459.

IPPUC. 1991. Histórico de Dados do Município de Curitiba. Curitiba, Instituto de Pesquisa e Planejamento Urbano de Curitiba, 163p.

Klein, R. \& G. Hatschbach. 1962. Fitofisionomia e notas sobre a vegetação para acompanhar a planta fitogeográfica do município de Curitiba e arredores (Paraná). Bol. Univ. Paraná, Geografia Física 4: 1-29.

Lamas, G. 1981. La fauna de mariposas de la Reserva de Tambopata, Madre de Dios, Peru (Lepidoptera, Papilionoidea y Hesperioidea). Rev. Soc. mex. Lep. 6 (2): 23-40.

LAMAS, G.; R.K. Robbins \& D.J. HaRveY. 1991. A preliminary survey of the butterfly fauna of Pakitza, Parque National del Manu, Peru, with a estimate of its species richness. Publ. Mus. Hist. Nat. UNMSA (A), Lima, 40: 1-19.

Mielke, O.H.H. \& M.M. Casagrande. 1991 (1992). Lepidoptera: Papilionoidea e Hesperiodea coletadas na Ilha de Maracá, Alto Alegre, Roraima, parte do Projeto Maracá, com uma lista complementar de Hesperiidae de Roraima. Acta Amazonica 21: 175-210.

Schaus, W. 1902. Descriptions of new American butterflies. Proc. U.S. Nat. Mus. 24: 383-460.

SEITZ, A. 1924. Die Gross-Schmetterlinge der Erde 5, Stuttgart, A. Kernen Verlag, 1141 p.

Recebido em 25.VIII.1994; aceito em 01.XII.1994. 